\title{
Management of Complicated Nasal Defects
}

\author{
James Owusu, MD ${ }^{1,2}$ Blaine Nesbitt, MD ${ }^{3} \quad$ Kofi Boahene, MD
}

${ }^{1}$ Department of Otolaryngology - Head and Neck Surgery, Mid-Atlantic Permanente Medical Group, McLean, Virginia

2 Department of Surgery, Uniformed Services University, Bethesda, Maryland

${ }^{3}$ Department of Otolaryngology - Head and Neck Surgery,

Uniformed Services University, Bethesda, Maryland

${ }^{4}$ Department of Otolaryngology Head and Neck Surgery,

Johns Hopkins Medical Institute, Baltimore, Maryland

Facial Plast Surg 2020;36:158-165.
Address for correspondence James Owusu, MD, Department of Head and Neck Surgery, Mid-Atlantic Permanente Medical Group, 8008 Westpark, Dr. McLean, VA 22102 (e-mail: owusuja@gmail.com).

\author{
Abstract \\ Keywords \\ - nasal reconstruction \\ - total nasal defect \\ - forehead flap \\ - full-thickness nasal \\ defects
}

The nose is the most common site for nonmelanoma skin cancer. Fortunately, most skin cancer lesions on the nose are discovered early and their timely diagnosis and excision result in superficial defects that can be reconstructed with minimal impact on the aesthetics and function. Reconstruction of full-thickness defects of the nose remains a challenging endeavor for reconstructive surgeons. An organized and systematic approach is necessary to streamline the analysis, planning, and reconstruction complex nasal defects to consistently achieve optimal results. This article reviews options for reconstructing full-thickness nasal defects and highlights current advances in established techniques.
The nose is a central aesthetic feature of the face and key in establishing self and familial identity. Studies using eye-tracking technology show that observers track the human face in a stereotypical pattern focusing on the central facial triangle. ${ }^{1}$ This characteristic visual scan path is distorted by distracting abnormalities of the central face such as nasal defects. Consequently, patients with central facial defects can have challenging social interactions because of distracted eye to eye contact. The principal goal of nasal reconstruction is to restore a functional nose that is aesthetically normal. Experience with nasal reconstruction has evolved over several centuries with each century adding a level of sophistication. Despite the accumulated experience, reconstructing a three-dimensional (3D) multilayer nasal defect, particularly those involving tip and ala with consistently normal aesthetics and functional outcomes remains a challenge. For the purposes of focused analysis, we define complex nasal defects as those involving all layers of the nose. Over the past three decades, there have been significant advances toward reliably reconstructing complex nasal defects. These advances have resulted from experience accumulated from critical analysis of poor outcomes and modification of techniques to address misjudgments and com-

plications. This article reviews principles, concepts, and evolving advances for reconstructing full-thickness nasal defects from subunits defects to extended total nasal defects.

\section{Clinical Presentations}

The nose is the most common site for nonmelanoma skin cancer due to its prominent location and vulnerable exposure to ultraviolent rays. ${ }^{2}$ Fortunately, most nasal lesions are discovered early and their timely excision results in small and often superficial defects that can be reconstructed with minimal impact on function and aesthetics. Resection of advanced and more aggressive neoplasms results in larger and complex multilayered defects that may extend beyond the boundaries of the nose and are challenging to reconstruct. In such cases, it is essential to ensure complete disease clearance prior to initiating the reconstructive process. This may require delaying the reconstruction until permanent pathologic reports are available and excising additional margins if necessary. A temporary application of adhesive-retained prosthetic noses should be considered when a long waiting period is

Copyright (C 2020 by Thieme Medical Publishers, Inc., 333 Seventh Avenue, New York, NY 10001, USA. Tel: +1(212) 760-0888.
Dol https://doi.org/ 10.1055/s-0040-1709141. ISSN 0736-6825. 
contemplated. The long-term use of implant-anchored prosthesis is also an alternative.

Complex nasal defects in children present a particular challenge beyond the technical aspects of reconstruction. Common causes of complex nasal defects in children include vascular malformations and dog bites. Congenital capillary hemangioma extensively involving the nasal tip, even after regression, leaves a deformed fibrous nose that often requires reconstruction. Dog bite injuries in children commonly involve complete avulsion of the nasal tip. The timing for reconstructing a nose in a child, the selection of less involved temporizing measures with skin grafts and local flap versus embarking on full scale complex reconstruction with forehead flaps are forefront in deliberations around the management of such defects. Burget detailed his experience over several years on the feasibility, outcomes, and long-term follow-up in 29 children who underwent complex nasal reconstruction using forehead flaps. ${ }^{3}$ In that series complex reconstruction began as early as 3.5 years and the reconstructed noses grew into adultsized noses, although additional delayed cartilage grafting was necessary in some patients.

\section{Principles and Concepts of Complex Nasal Reconstruction}

It is broadly established that complex nasal defects involving the nasal skin envelope, supportive substructure, and nasal lining require replacement and reconstruction of all three layers without compromise. The donor skin should provide excellent color and texture match and should reflect the contours of a normal nose. The supportive substructure should be stable from displacement, maintain nasal patency, and withstand secondary contraction from inevitable scarring. The nasal lining should be thin to maintain nasal patency and well vascularized to support overlying grafts. Beyond these foundational principles, several concepts that can guide complex nasal reconstruction have evolved.

1. Defects involving large portions of subunits, particularly the convex contours of the nasal tip, are best resurfaced as a whole unit.

2. It is advantageous to delay cartilage grafting till a wellvascularized lining has been established.
3. More nasal lining than imagined is often necessary to maintain nasal patency.

4. Skin grafts supported by vascularized forehead flaps are reliable sources of nasal lining.

5. The donor tissues, sequence of surgeries, and the timing should permit intraoperative modification, opportunities to revise imperfections, or salvage complications. A three to four-stage procedure is often necessary for complex nasal defects.

6. There is benefit in delaying division of the forehead flap pedicle until all intermediate steps including soft tissue partition, thickness modification, and 3D contouring have been satisfactorily completed.

7. Small salvage steps often yield small unsatisfactory results. A revision case may require starting all over.

\section{Defect Analysis and Planning}

Successful reconstruction of a complex nasal defect starts with a thorough assessment of the defect to establish the layers involved and extent of tissue missing. An organized and systematic approach is necessary to streamline the analysis, planning, and reconstruction to achieve optimal results with consistency. The entire nose, both external and internal, should be carefully examined. The quality, vascularity, and mobility of surrounding skin and supporting structures should be evaluated to establish where donor tissue could be recruited from without distorting adjacent structures. Based on the extent of tissue involved, fullthickness nasal defects can be subclassified as (-Fig. 1):

A. Complex subunit defect involves all layers of one subunit.

B. Complex subtotal defect involves more than one subunit; caudal septum is present.

C. Total nasal defect involves multiple subunits and caudal septum with or without nasal bone involvement.

D. Extended total nasal defect; total nasal defects as above with involvement of cheek or lip.

Defining the presented defect, after resection of scar tissue and expansion of the defect into subunits allows a tailored approach into solving the puzzle. An inside-out approach beginning with evaluation for lining, followed by
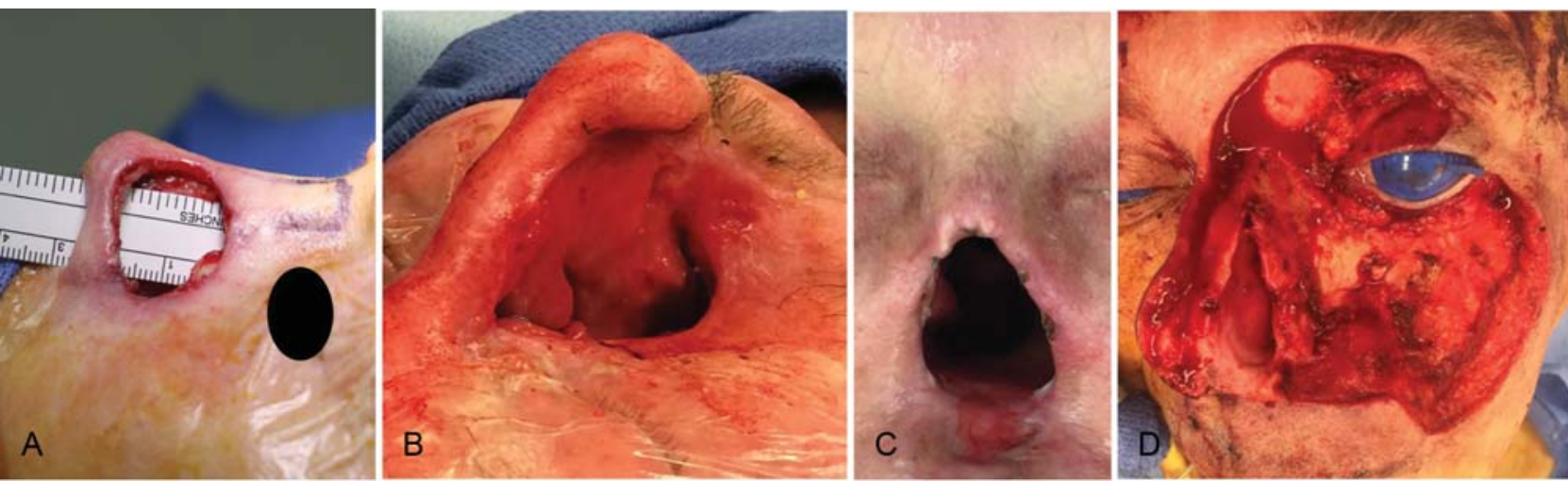

Fig. 1 Subclassification of complex nasal defects. (A) Complex subunit defect. (B) Complex subtotal defect. (C) Total nasal defect. (D) Extended total nasal defect. 
structural needs and then external soft tissue coverage, forces the surgeon to avoid focusing only on the obvious surface tissue loss.

\section{Reconstructing the Nasal Lining}

The nasal lining is the least visible portion of a complex nasal defect but perhaps the most important aspect of the reconstruction. The nasal cavity is lined with squamous epithelium and respiratory epithelium that filters and conditions inspired air. The vibrissae filter large particles, while the moist mucosal lining traps smaller particles and provides humidification. A well-vascularized nasal lining is critical for successful complex nasal reconstruction. Suboptimal lining with gaps leads to graft exposure, infections, undesirable contractions, and ultimately reconstruction failure. An adequately vascularized lining is necessary to support structural grafts. The ideal lining should be thin to allow airway patency. In addition, maintaining maximal humidification and filtration of inspired air is desirable to minimize intranasal crusting of the reconstructed nose. Options for reconstructing lining defects include grafts, local flaps, regional flaps, and microvascular tissue transfer.

Free grafts have limited role in the reconstruction of fullthickness nasal defects unless a vascularized supportive bed can be provided. A commonly used free graft is the composite skin-auricular cartilage graft. These are useful for small defects isolated to the alar subunit. Small windows can be made through the cartilage to allow vascularization of the adherent skin. ${ }^{4}$ The main advantages of the composite auricular skin cartilage graft are their favorable contour and the simultaneous introduction of thin lining skin adherent to a supporting cartilage. These grafts should be avoided in children as they rarely provide adequate support and growth over time.

Hinged turn-in skin flaps recruited from subunits adjacent to the defect to be repair are also alternative sources of lining. ${ }^{5}$ While these may be partially vascularized, their distal end should be considered as free grafts requiring a good vascularized bed for reliable survival. Hinged turn-in skin flaps are rarely adequate sources of lining and are often combined with additional sources of lining. While it is tempting to stretch these turn-in flaps, this leads to tenting and secondary contraction. Turn-in flaps that may have appeared to be acceptable lining initially contracts over time and becomes inadequate coverage to maintain a patent intranasal vault. Another source of skin for nasal lining is the distal tip of a forehead flap as described by Menick. ${ }^{6}$ The designed lining is incorporated as an extension of the distal edge of the forehead flap to draped internally beyond the alar and nostril margin. Once the folded skin has integrated into the adjacent normal lining, it can be completely separated from the overlying cover from which it was initially vascularized. The folded forehead flap is incised free along the rim, completely separating the proximal cover flap from the distal lining extension. Supporting and contouring cartilage grafts can then be placed.

Another source of intranasal lining is nasal mucosa. Various mucosal flaps have been described for small and intermediate sized defects. These include turbinate mucosal flaps, bipedicled vestibular flap, ipsilateral and contralateral septal flaps. ${ }^{7,8}$ Intranasal mucosa is an attractive source of lining because it replaces like tissue, it is adjacent to the nasal defect, and has the potential of providing a moist and humidified reconstructed nose. There is, however, a finite amount of nasal mucosa limiting their use when defects are large. When available, one versatile way to recruit septal mucosa for inner lining is the transposition of the septum as a composite bilateral mucosal-cartilage flap (septal pivot flap) pedicled on the septal branch of the superior labial artery. The septal pivot flap was described by Burget and Menick in $1989^{9}$ and its application in complex nasal reconstruction was also detailed by Quetz. ${ }^{10,11}$ The septal branch of the superior labial artery is likely preserved if the nasal defect is limited to the upper half of the caudal septum and the central upper lip is uninvolved. The pair of septal branches of the superior labial artery allows almost the entire septum to be elevated as a bimucosal cartilage composite flap after a superior, posterior, and inferior septal release. At least a 12-mm wide intact mucosal bridge should be preserved inferiorly as a pedicle. With the composite flap transposed, the cartilage provides caudal septal replacement and support for the nasal tip and the mucosa can be elevated bilaterally to line the nasal tip and vestibule.

\section{Laminated versus Prelaminated Forehead Flap}

An extension of the use of hinged skin flaps and small skin grafts sutured to mucosal flaps is the use of skin graft to replace all the needed lining. The primary attraction for this approach is the unlimited supply of skin graft. The skin graft requires a vascularized bed, which is provided by a full-thickness forehead flap. The forehead flap is elevated with all the layers above the periosteum. For this approach to be successful, the skin graft needs to be immobilized to the undersurface of the forehead flap till the skin becomes fully vascularized. In the prelaminated approach, a split-thickness skin graft (STSG) is harvested and sutured to the undersurface of the elevated forehead flap. ${ }^{12}$ The graft is sutured to the edge of the forehead flap as well as with central tucking sutures (-Fig. 2). The STSG is oversized forming some rugae; these smooth out with contraction. The forehead flap with the STSG is replaced and sutured back on the forehead. The frontal bone acts as a compressive bolster and thus an external bolster is not necessary. Potential complication with this approach is seroma formation, which is treated with needle decompression. In cases where postoperative radiation is anticipated, the forehead flap is elevated at the time of primary tumor resection and prelaminated. We have left the prelaminated forehead flap in place as long as 6 months to allow completion of radiation. The forehead flap is usually out of the radiation field. This approach saves the patient one operation, allows contraction of the forehead flap to occur and stabilize prior to flap transfer, and yields a well-vascularized lining.

Alternatively, STSG may be applied in a laminated approach. With the laminated approach, the STSG is sutured to the edges of the nasal defect accounting for the anticipated nasal 


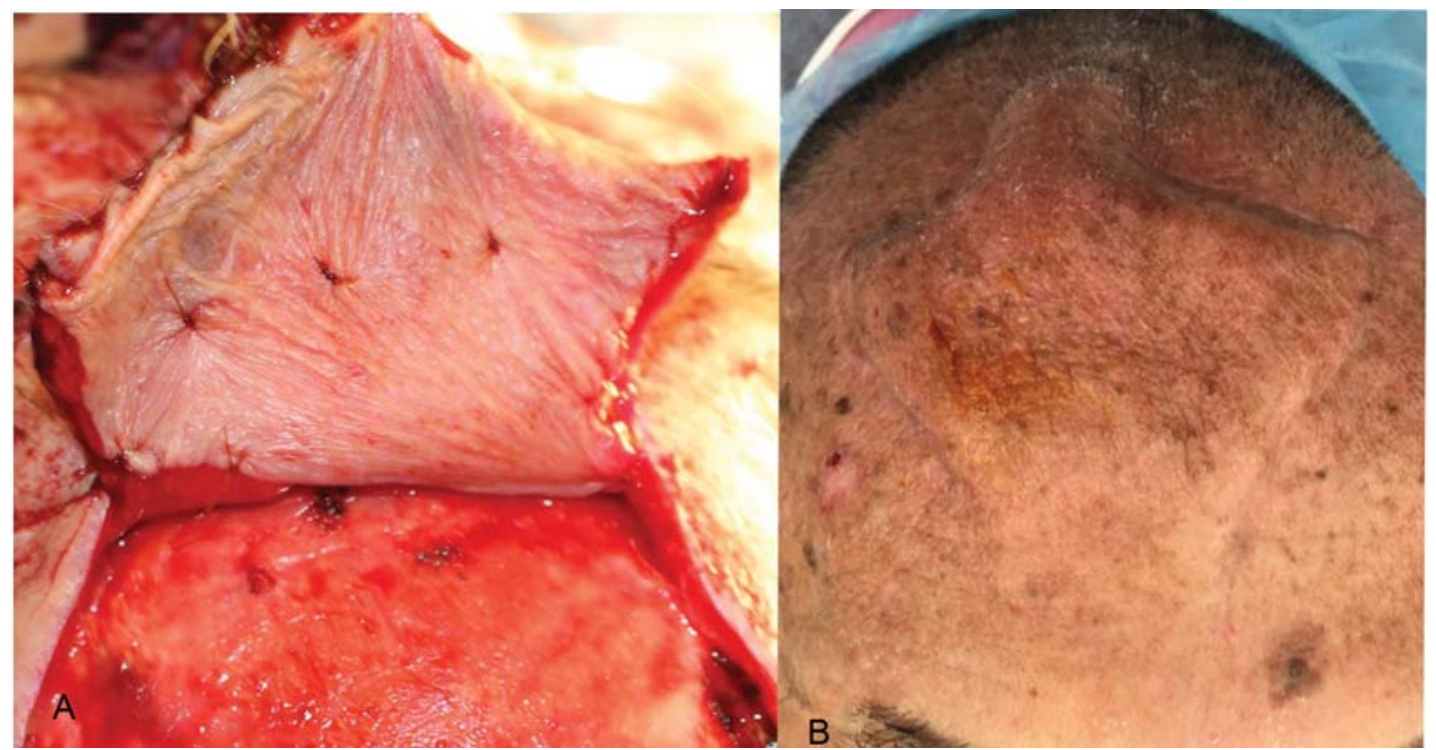

Fig. 2 Prelaminated forehead flap. (A) Split-thickness skin graft is sutured to the undersurface of a forehead flap elevated in a plane above the periosteum. The skin graft is made slightly loose with some rugae that smooths out with secondary contraction. (B) The composite flap is elevated after a minimum or 3 to 4 weeks and transposed to the nasal defect. At an intermediary stage, the forehead skin is elevated in a subcutaneous plane leaving the skin graft, galea and frontalis muscle as the new vascularized lining.

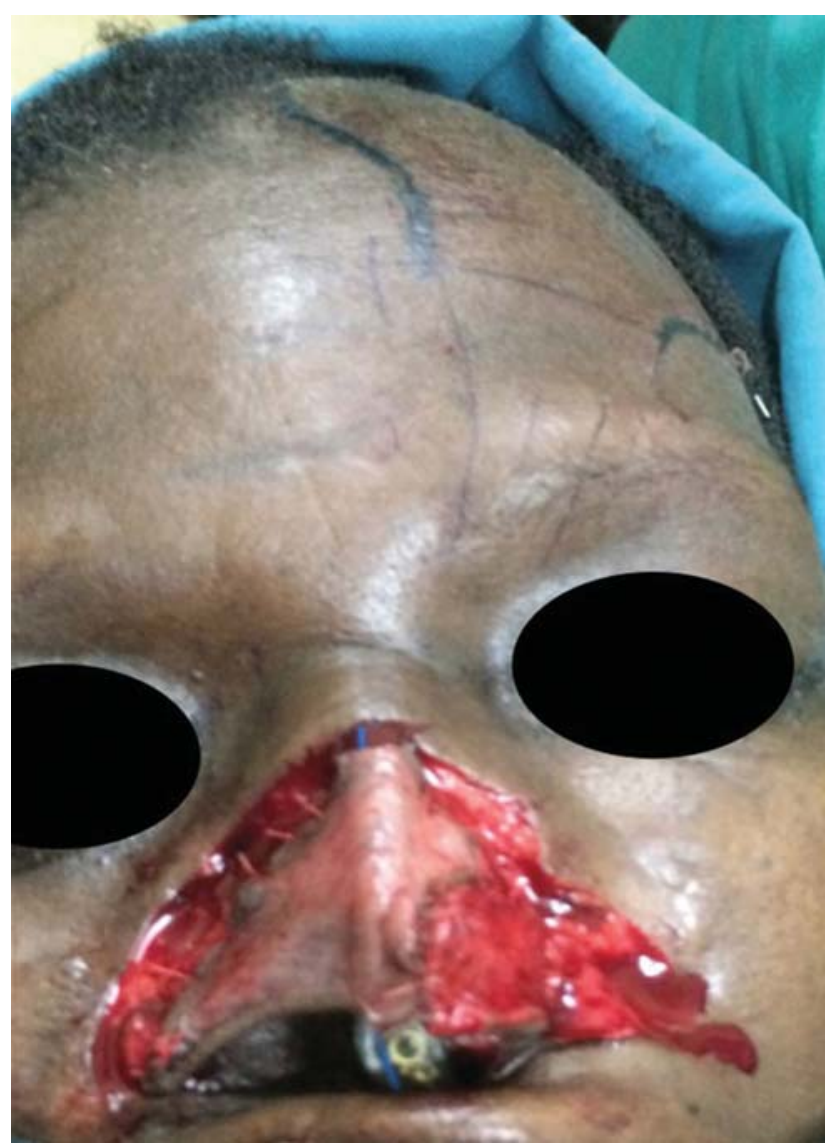

Fig. 3 Laminated forehead flap. Split-thickness skin graft is sutured to the edges of the nasal defect and supported by a bolster packing. The forehead flap is then harvested above the plane of the periosteum and transposed over the skin graft lining. The forehead skin can be elevated 3 to 4 weeks later in a subcutaneous plan leaving the skin graft, galea and frontalis muscle as the new vascularized lining. projection (-Fig. 3). The forehead flap is then elevated and transposed to provide external coverage of the nasal defect. An intranasal bolster is necessary to support and immobilize the STSG against the undersurface of the full-thickness forehead flap. Options for intranasal bolster include cotton packed to support the STSG and to act as an absorbent or packed petroleum gauze. We have also found a modified inflated low-pressure endotracheal tube very effective. The cuff conforms to the intranasal space and is easily deflated and reinflated to inspect the STSG.

With either the prelaminated or laminated approach, an intermediate stage is performed to split the forehead flap elevating the entire flap in a subcutaneous plane. The nasal lining consisting of the STSG, galea, and some frontalis muscle is now vascularized from surrounding vessels as well as the forehead pedicle. The pedicle is kept intact, the lining is modified as needed, and cartilage grafts are placed (-Fig. 4). The use of prelaminated and laminated forehead flap has altered our approach to complex nasal reconstruction. We now consider this our primary approach for providing nasal lining leaving native nasal mucosa in place to maximize their humidifying function. With either the prelaminated or laminated approach, secondary contraction in the transposed flap should be anticipated and accounted for in the design of the flap and lining by slightly over-sizing. We harvest the STSG from the chest wall in line with the incision for future rib cartilage graft harvest and consequently avoiding a second donor site scar.

The free transfer of tissue can provide an abundant tissue for lining and should be considered as primary source in selected cases. We consider these procedures low morbidity procedures when performed by experienced surgeons and not an option of last resort. Microvascular flaps are particularly useful in extended total nasal defects where the 


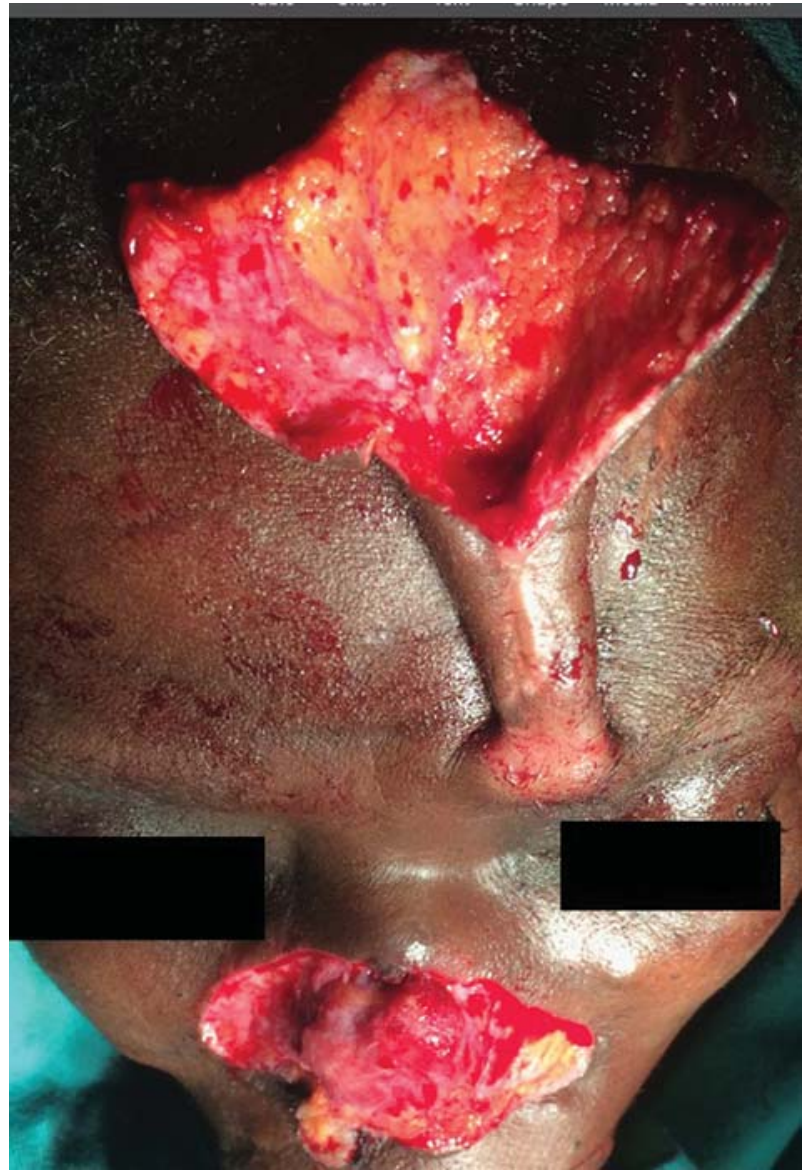

Fig. 4 Separating the lining: 3 to 4 weeks following a insertion of the composite forehead flap with skin graft lining, a robust vascularized lining made of the skin graft, galea and frontalis muscle results. The new lining is vascularized from the surrounding tissues and from the intact forehead flap pedicle. It can be thinned as needed.

foundation of the nose at the maxillary and premaxillary buttresses is involved. In addition, involvement of the upper lip and extensive segment of the medial cheek soft tissue necessitates the transfer of distal skin best achieved with free flaps.

The anterolateral thigh (ALT) and radial forearm free flaps are the two most common free-flap options for reconstructing internal lining defects. The ALT flap is commonly harvested as a fascia flap and provides thin vascularized tissue to reconstruct the entire nasal lining and adjacent structures with minimal donor site morbidity. The ALT fascia becomes secondarily mucosalized. ${ }^{13,14}$ The radial forearm free flap also provides enough tissue for reconstructing the lining in total nasal defects. The radial forearm free flap can be harvested with skin or as a fascial flap with secondary mucosalization. ${ }^{15}$

\section{External Coverage}

The nasal skin varies in thickness and texture when one transitions from the upper to lower thirds of the nose. Together with the underlying layer of subcutaneous fat and superficial musculo-aponeurotic system, the nasal skin reflects the contours of the underlying cartilage and bony substructure. The soft tissue envelope has been divided into topographic subunits that reflect or absorb light differentially to provide nasal definition. The subunit principle proposed by Burget and Menick when resurfacing nasal defects recommends replacing an entire subunit when more than $50 \%$ of the subunit is missing. The subunit principle is mainly applicable to defects of the nasal tip and alar subunits. ${ }^{16}$ Because of their convex contour, defects that are irregularly shaped and occupy more than $50 \%$ of the tip or ala subunit are best expanded and replaced as an uninterrupted unit for a uniform contour and textural match.

Defects of the external soft tissue envelope should be viewed in four dimensions: length, width, thickness, and time anticipating and factoring secondary changes from contraction into the design of templates. For unilateral defects, the ideal template is created from the contralateral side. Templates created directly from the defect are less accurate as edema and wound contraction will likely distort the size of the wound. For bilateral defects and more extensive near total nasal defects, additional aids in obtaining accurate templates may be necessary. Facial surface scanning technology can be helpful in generating stereolithography data for 3D models of the nose that can be fabricated by an anaplastologist or 3D printed for intraoperative use. 3D surface scanning is helpful when the nose is not overly distorted by cancer prior to resection. Medical artists can be helpful in generating idealized drawings of a patients nose from which prosthetic models can be generated, scanned, and manipulated for template generation (-Fig. 5). The technology for surface scanning has become ubiquitous. There are currently several 3D scanning applications on the market for smartphones and tablets. Once an accurate 3D model is obtained, a 2D template is generated from which the desired skin for precise reconstruction can be determined and outlined on the donor site. While 1:1 tissue replacement of missing skin over the dorsum, tip, and sidewalls

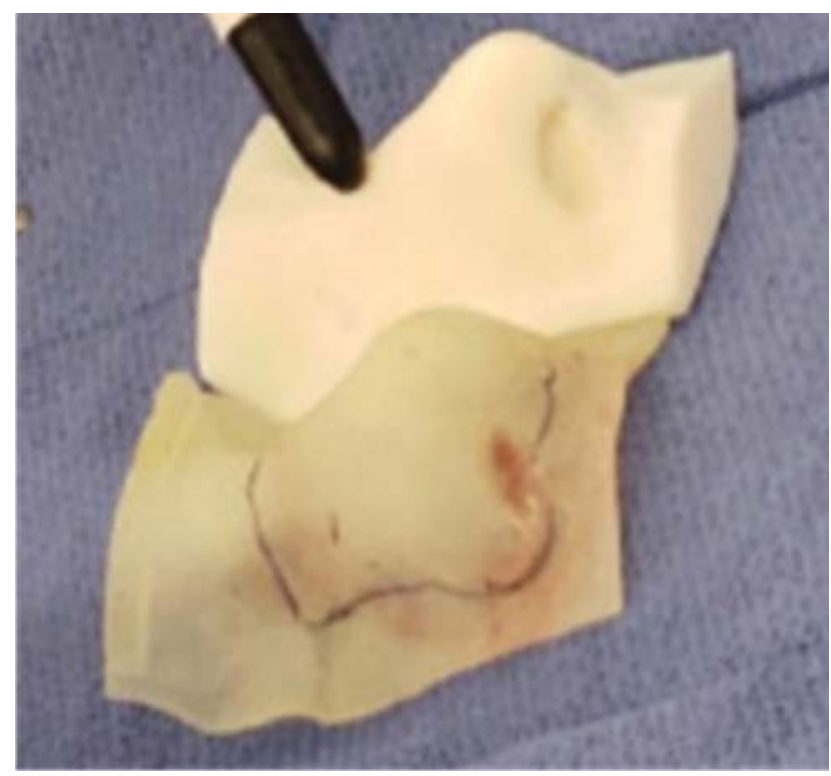

Fig. 5 Three-dimensional models are particularly helpful in total nasal defects in determining the needed lining, projection, and in designing two-dimensional templates for the forehead flap. 


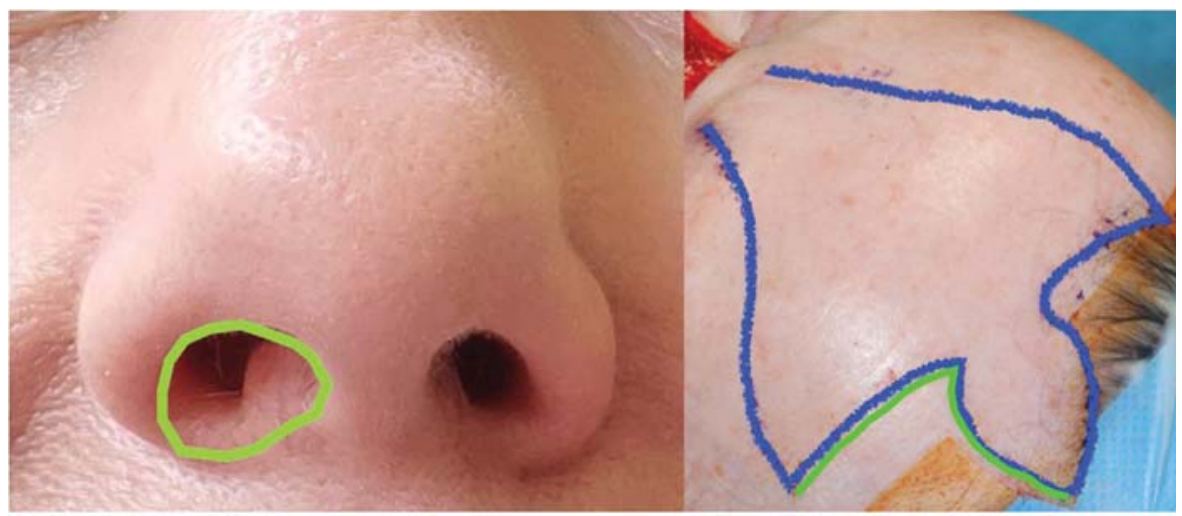

Fig. 6 The forehead flap design should account for the true circumference of the nostrils (green lines) and should not be compromised to reduce the size of the forehead defect.

is ideal, a calculated overcorrection may be prudent when designing the 2D template for a circumferential nostril defect. Templates designed to include the nostril should account for the nasal sill (-Fig. 6). A common complication of complex nasal reconstructions is a stenotic nostril that often fails to respond to stenting. The use of a $2 \mathrm{D}$ flap to reconstruct a $3 \mathrm{D}$ defect is not straightforward and entails variables made evident by time, the fourth dimension, wound healing, and contraction and skin compromise. Consequently, it is prudent to stage these procedures allowing room for modifications.

The forehead flap remains the workhorse flap for reconstructing large external nasal soft tissue envelope defects. The forehead flap provides ample vascularized tissue with excellent color and texture match and acceptable donor site morbidity. There is no good alternative to the forehead flap so bridges should not be burned in their design. In rare cases where the forehead skin is unavailable for reconstruction, a radial forearm free flap is an alternative option for reconstructing the nasal envelope. The color match for free flaps is usually poor.

\section{Classic Design of the Forehead Flap}

Forehead flaps can be harvested as a paramedian flap or midline flap based on the supratrochlear vessels and the central artery. The extensive vascularization of the forehead allows for the harvest of two to three flaps from the same side. ${ }^{17}$ Intraoperative angiography can be used in assessing vascularity of the forehead flap in revision cases when available.

The classic forehead flap is harvested as a paramedian flap based on the supratrochlear artery. Pearls for designing this flap include limiting the pedicle base width to $1.5 \mathrm{~cm}$ and designing the flap as vertical as possible and not greater than 15 degrees off vertical. ${ }^{3}$ The size of the forehead flap should not be compromised to save forehead skin. Very large donor site defects can be narrowed with spanning or purse string suturing and residual defect allowed to heal by secondary intention. Efforts should be made to minimize transfer of hair-bearing scalp to the nose but not at the expense of shortening the reach of the flap. Techniques that increase the reach of the flap reduce the risk of transferring hair-bearing scalp. For lateral defects, an ipsilateral flap has a longer reach compared with a contralateral flap. The reach of the flap can also be increased by extending the medial flap incision below the level of the brow. Careful transverse release of the frontalis muscle across the width of the flap can gain $\sim 1 \mathrm{~cm}$ of effective length.

The forehead flap can also be designed as a midline flap based on the central and paracentral arteries. The pedicle to the midline forehead flap can be oriented slightly obliquely and extended along the nasal side wall between the medial canthus and the nasal dorsum extending the reach of the flap. This design is helpful in patients with a low hairline. ${ }^{18}$

In patients with very low hairline or deficient skin as a result of previous surgery, expansion of the forehead skin can be considered. ${ }^{17,19}$ Expanded forehead flaps should be considered as a truly last resort since expansion can compromise the dense subcutaneous fat of the forehead skin that makes it an ideal donor site for nasal reconstruction.

\section{Nasal Foundation}

A stable foundation is required to support the reconstructed nose. The nose is supported by the nasal bones and its articulation with the frontal bone and frontal processes of the maxilla. The midline intranasal support by the perpendicular plate of the ethmoid bones and the quadrangular septal cartilage is critical for maintaining intranasal patency. The medial maxillary buttresses as well as the premaxillary bone with their respective overlying soft tissues (cheek and lip) are essential transitions of the nose. Defects that extend beyond the nose to involve the cheek and lip soft tissues require additional attention without which the stability of the reconstructed nose becomes secondarily compromised (-Fig. 1D). To obtain a predictable result, deep cheek and lip defects may need to be reconstructed and allowed to stabilize before reconstructing the nasal defect. Reconstructing the nose and surrounding facial subunit defects in a single stage produces unpredictable outcomes secondary to contraction of the cheek or lip repair.

\section{Nasal Framework}

The intricate 3D appearance of the nose reflects the underlying framework. The nasal framework is a composite of the rigid bony vault comprising the paired nasal bones and the 
frontal processes of the maxilla. Overlapping and articulating to the bony pyramid are the upper lateral cartilages and the nasal septum. The shape and support of the nasal tip is a result of the paired lower lateral cartilages and their interaction with the nasal spine, caudal septum, supporting ligaments and the resilience of fibrous tissue of the free alar margins. Without adequate and durable framework replacement, any elaborate nasal reconstruction is likely to fail. Reconstruction of the nasal framework can be anatomic and nonanatomic and should be approached with the mindset of a structural engineer. Rigid grafts should be placed to counter retraction, twisting and tilting in response to scar contraction. Anatomic grafts replace the named structures of the nose: septum, upper lateral cartilage, and nasal bones. Nonanatomic grafts are those grafts placed in areas that are naturally devoid of rigid support such as alar rim grafts and cartilage grafts placed between the piriform margin and the alar crease to minimize contraction and retraction. In general, it is best to repair bone defects with bone graft and cartilaginous defects with cartilage, although replacing one tissue type with the other is feasible.

When absent, reconstruction of the caudal septum with appropriate projection and stability is critical. As described earlier, a septal pivot flap is effective in transposing septal cartilage from the posterior septum anteriorly to provide caudal septal support. In the absence of septal cartilage, the forehead flap can be designed with a wide columella segment to wrap around a caudal septal strut.

Autologous bone and cartilage grafts are the ideal options for reconstructing nasal framework defects. Common donor sites include auricular cartilage, septal cartilage, septal bone, costal cartilage, and split calvarial bone grafts. Radiated rib cartilage grafts may also be used, although they introduce an element of uncertainty as to their long-term stability, increased risk for resorption or infection. The choice of donor graft material depends on the location and extent of the defect. For instance, the natural curvature of the concha cartilage makes it an ideal option for the reconstruction of alar defects. In general, rib cartilage graft is preferred to auricular cartilage graft in both children and adults as it provides ample cartilage with durable strength. In children in particular, auricular cartilage does not appear to provide durable support and lag in growth. ${ }^{3}$

The timing of framework reconstruction is an important consideration when planning for complex nasal defect recon- struction. For structural grafts to be effective, they must remain well vascularized over time. As such, it is recommended to defer the placement of structural grafts to the intermediate stages when the soft tissue envelope and lining have become well vascularized. A temporary structural support using titanium mini plates may be used in such cases to prevent excessive contraction and distortion of the soft tissue construct.

The cartilage graft applied may be considered as structural versus contouring graft. The central and lateral structural grafts are placed during the intermediate steps after the nasal lining has stabilized. Central structural grafts include columella struts, caudal septal graft, dorsal onlay grafts, extended spreader grafts; these reconstitute the support L-strut and provide the needed projection of the nose. The lateral structural grafts include the alar batten grafts, rim graft, and nonanatomic graft placed between the nasal bones and alar batten grafts (-Fig. 7).

Alar battens are best sculpted from strips of rib cartilage graft. These strips warp when carved but may be modified further with sutures and strut grafts to take on the desired shape. The alar battens graft should have the shape of typical alar lobule and should extend into the soft triangle region to give support to the nostril margin. ${ }^{11}$

\section{Contouring and Refinement}

To achieve a defined nose, the skin envelope must reflect light over the dorsum and absorb light along the alar crease, supra tip, and nasal sidewall. This requires an appropriately thinned skin envelope and strategically placed contour graft. Creation of the alar crease seems to be elusive. Burget and Menick $^{3,19}$ had proposed full-thickness skin incision with anchoring sutures to aid in alar crease formation. We have found the use of subcision with a 2-mm biopsy punch very effective in creating an alar crease.

The nostrils should be inspected at this stage for patency and symmetry. Minor skin excision and tissue rearrangement techniques are employed to even out any asymmetries.

\section{Pedicle Division}

Pedicle division is reserved as the last step in these complex nasal reconstructions and is performed only when a stable and satisfactory nose free of complications has been achieved.

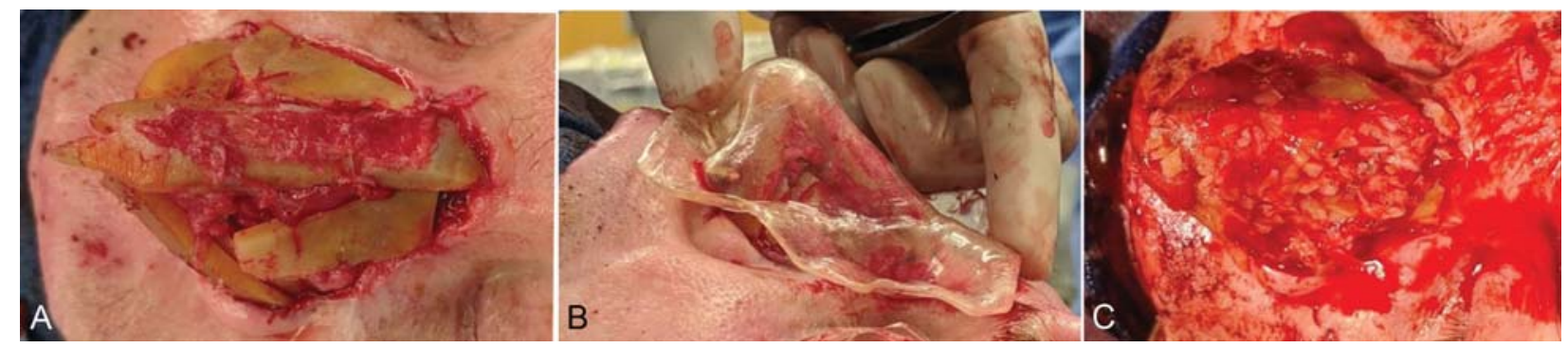

Fig. 7 Structural grafts should include stable central and transverse columns. These include anatomic and nonanatomic grafts (A). Additional contour grafts are placed as onlay grafts to fill in all the spaces between the structural grafts. Here diced cartilage stabilized with fibrin glue is contoured into place with the aid of a transparent three-dimensional model of the desired nose (B, C). 


\section{Smokers}

It is well known that smoking has a detrimental effect on wound healing. Smoking is associated with tissue hypoxia and thrombosis of the microvasculature. This physiologic impact of smoking results in skin necrosis, increased risk of infection, and wound dehiscence. ${ }^{20}$ Smoking cessation should be recommended, and increased risk of complications should be discussed preoperatively with patients who are active smokers. There is an increased risk of failure when random flaps and composite grafts are used in active smokers; these techniques should be avoided in smokers whenever possible.

\section{Conclusion}

Techniques for nasal reconstruction have been refined over several decades. Current techniques allow reconstruction of most complex defects with normal functional and aesthetic outcomes. With careful planning and meticulous execution consistently, good results can be achieved.

\section{Conflict of Interest}

None.

\section{References}

1 Dey JK, Ishii LE, Byrne PJ, Boahene KDO, Ishii M. Seeing is believing: objectively evaluating the impact of facial reanimation surgery on social perception. Laryngoscope 2014;124(11): 2489-2497

2 Conte CC, Razack MS, Sako K. Skin cancer of the nose: options for reconstruction. J Surg Oncol 1988;39(01):1-7

3 Burget G. Aesthetic Reconstruction of the Child's Nose. Chicago, IL: Gary Burget; 2012

4 Park SS. Nasal reconstruction in the 21st century-a contemporary review. Clin Exp Otorhinolaryngol 2008;1(01):1-9
5 Park SS, Cook TA, Wang TD. The epithelial 'turn-in' flap in nasal reconstruction. Arch Otolaryngol Head Neck Surg 1995;121(10): $1122-1127$

6 Menick FJ. Nasal reconstruction with a forehead flap. Clin Plast Surg 2009;36(03):443-459

7 Murakami CS, Kriet JD, Ierokomos AP. Nasal reconstruction using the inferior turbinate mucosal flap. Arch Facial Plast Surg 1999;1 (02):97-100

8 Menick FJ. Nasal reconstruction. Plast Reconstr Surg 2010;125 (04):138e-150e

9 Burget GC, Menick FJ. Nasal support and lining: the marriage of beauty and blood supply. Plast Reconstr Surg 1989;84(02):189-202

10 Quetz J. Update on the septal pivot flap. Facial Plast Surg 2014;30 (03):300-305

11 Quetz J, Ambrosch P. Total nasal reconstruction: a 6-year experience with the three-stage forehead flap combined with the septal pivot flap. Facial Plast Surg 2011;27(03):266-275

12 Gassner H, Sadick H, Haubner F, Artinger V, Kuehnel T. Prelamination to reconstruct internal nasal lining. Facial Plast Surg 2013; 29(05):411-416

13 Revenaugh PC, Haffey TM, Seth R, Fritz MA. Anterolateral thigh adipofascial flap in mucosal reconstruction. JAMA Facial Plast Surg 2014;16(06):395-399

14 Seth R, Revenaugh PC, Scharpf J, Shipchandler TZ, Fritz MA. Free anterolateral thigh fascia lata flap for complex nasal lining defects. JAMA Facial Plast Surg 2013;15(01):21-28

15 Winslow CP, Cook TA, Burke A, Wax MK. Total nasal reconstruction: utility of the free radial forearm fascial flap. Arch Facial Plast Surg 2003;5(02):159-163

16 Burget GC, Menick FJ. The subunit principle in nasal reconstruction. Plast Reconstr Surg 1985;76(02):239-247

17 Park SS. The single-stage forehead flap in nasal reconstruction: an alternative with advantages. Arch Facial Plast Surg 2002;4(01):32-36

18 Faris C, van der Eerden P, Vuyk H. The midline central artery forehead flap: a valid alternative to supratrochlear-based forehead flaps. JAMA Facial Plast Surg 2015;17(01):16-22

19 Menick FJ. A 10-year experience in nasal reconstruction with the three-stage forehead flap. Plast Reconstr Surg 2002;109(06): 1839-1855, discussion 1856-1861

20 Little SC, Hughley BB, Park SS. Complications with forehead flaps in nasal reconstruction. Laryngoscope 2009;119(06):1093-1099 